\title{
the Action of Cyanotoxin $\beta-N$-Methylamino-L-Alanine (BMAA) on the Key Cellular Processes in Diazotrophic Cyanobacteria
}

\author{
O. A. Koksharova 1,2, I. O. Butenko ${ }^{3}$, O. V. Pobeguts ${ }^{3}$, N. A. Safronova ${ }^{1}$, and V. M. Govorun ${ }^{3}$
}

'Lomonosov Moscow State University, Belozersky Institute of Physico-Chemical Biology, Leninskie Gory, 1-40, Moscow, 119991, Russia;

2Institute of Molecular Genetics of National Research Center "Kurchatov Institute", Kurchatov Square, 2, 123182 Moscow, Russia

${ }^{3}$ Scientific-Research Institute of Physical-Chemical Medicine, Moscow 119435, Russia;

*Correspondence: koksharova@genebee.msu.ru; Tel.: +7-917-534-7543

\section{Introduction}

Cyanobacteria are the oldest photoautotrophic microorganisms. In order to supply all cells in cyanobacterial filaments with nitrogen in nitrogen-free growth condition diazotrophic cyanobacteria use mature heterocysts (Figure 1) that are specialized nitrogen fixing cells that convert atmospheric nitrogen into bioavailable nitrogen and transport it into neighbor vegetative cells; while vegetative cells provide heterocysts with carbon and reductants (Figure 2) [1]. Non-proteinogenic neurotoxic amino acid $\beta-\mathrm{N}$-methylamino-L-alanine (BMAA) (Figure 3) is a bioactive molecule synthesized by various phytoplankton species, such as: cyanobacteria, diatoms and dinoflagellates, and is known to be a causative agen of human neurodegeneration diseases [2]. It was found that the addition of BMAA inhibited nitrogenase activity in mature heterocysts of diazotrophic Nostoc sp. PCC 7120 [3,4]. The use of real-time PCR allowed the discovery of inhibited expression of a nitrogenase-specific gene, nifH, by BMAA in this cyanobacterium [4]. Furthermore, under nitrogen starvation, at the start of heterocyst differentiation, adding BMAA inhibited this process. BMAA

downregulates transcription of the key heterocyst-specific genes hetR and hepA in Nostoc [4]. An unusual opposite regulatory effect of BMAA on diazotrophic cyanobacteria was found in nitrogen-replete conditions [5]. Therefore, we were interested in the question: what kind of mechanisms underlie action of BMAA in cyanobacteria cells under different nitrogen supply conditions.

\section{Aim}

The aim of our proteomic study was to examine the biological effects of exogenous BMAA on diazotrophic cyanobacterium Nostoc sp. PCC7120 in different growth conditions

\section{Methods}

Cyanobacterial strain Nostoc sp. PCC 7120 and growth cultivation conditions in BG11 medium are described in [6-8]. The analysis was performed on a Triple TOF 5600+ mass spectrometer with a NanoSpray III ion source (AB Sciex, Framingham, MA, USA) coupled with a NanoLC Ultra 2D+ nano-HPLC system (Eksigent, now part of Sciex, Framingham, MA, USA). Identification of proteins was performed with ProteinPilot (version 4.5, Sciex, ABSciex, Forster, CA, USA). All LC-MS/MS data were searched against the National Center for Biotechnology Information (NCBI) GenBank protein sequence database for Nostoc sp. PCC 7120. Methods of Trypsin Digestion in Solution, LC-MS/MS Analysis, Protein Identification by LC-MS/MS Data Analysis and Pathway Analysis Based on LC-MS/MS Data are described in [6-8]

\section{Results}

Altogether, 1567 proteins of cyanobacterium Nostoc sp. PCC 7120 were identified [6-8]. Among them, dozens of proteins belonging to different functional categories were selected for further analysis and discussion based on the statistical significance of the observed differences between BMAA-treated and control samples in three growth conditions. Our proteomic studies have demonstrated that under BMAA-treatment the most significant difference lies in the expression change of a key nitrogen regulatory protein PII (Figure 4). This protein is downregulated in nitrogen-starving conditions and it is upregulated in nitrogen-replete conditions in the presence of BMAA [6,7]. This could be the main reason behind a specific regulatory effect on heterocyst formation and an abnormal heterocyst- and nitrogenase-related gene expression that is caused by this amino acid in Nostoc sp. PCC $7120[4,5] .$. The primary main targets of BMAA action in cyanobacteria cells are, apparently, metabolic processes, such as nitrogen fixation [6-8], photosynthesis, carbon fixation and different biosynthetic processes, the regulation of which involves 2-oxyglutarate and glutamate and proteins, whose gene transcription is under NtcA regulation (Figure 5) [8].. It can be hypothesized that BMAA may be used by phytoplankton representatives (cyanobacteria, diatom, dinoflagelates) as a possible allelopatic tool to control cyanobacteria cell populations during their competition for nitrogen and other resources (Figure 6) [8].

\section{Conclusions}

BMAA remarkably affects proteome expression of Nostoc sp. PCC 7120 in all tested growth conditions: during the heterocyst formation stage under nitrogen starvation conditions, in nitrogen replete conditions and during diazotrophic growth.

2) The action of BMAA leads to changes in nitrogen-carbon balance regulation that differs in cyanobacteria cells under nitrogen starvation and in nitrogen-replete conditions.

3) BMAA downregulates a key nitrogen regulatory protein PII and other proteins that are involved in nitrogen metabolism and heterocyst formation.

\section{Acknowledgments}

This work was supported by the Russian Foundation for Basic Research (grant \# 17-04-00412).
Filamentous cyanobacterium Nostoc (Anabaena) sp. PCC 7120
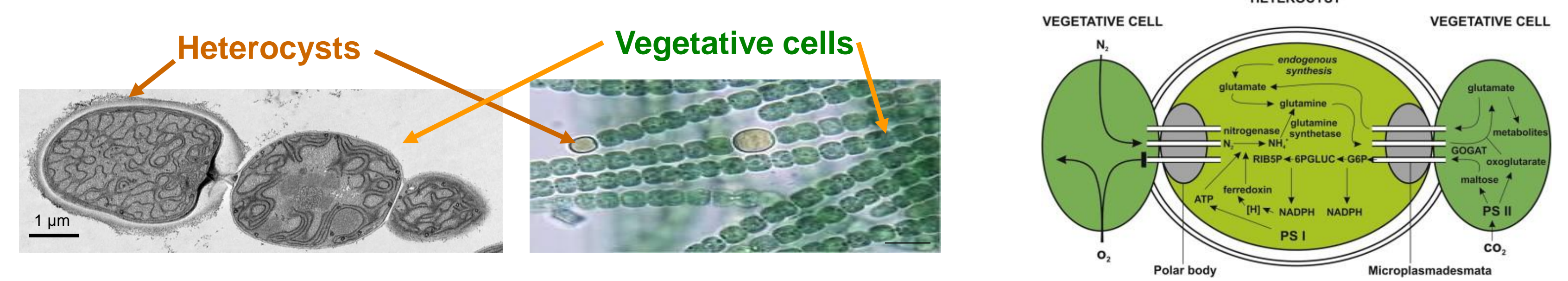

Fig.1. Two types of cells in cyanobacterial filaments: heterocysts and vegetative cells.

Fig.2. Heterocysts are specialized cells for nitrogen fixation [1]
Nitrogen-repete

conditions<smiles>CNC[C@H](N)C(=O)O</smiles>

$\beta$-N-methylamino-L-alanine (BMAA)

(a)

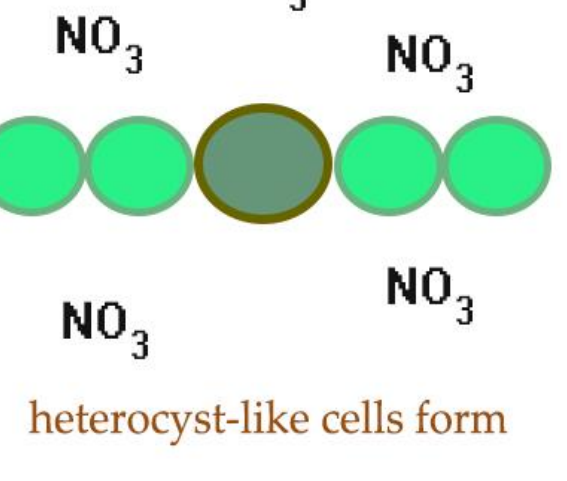

$\mathrm{PII} \uparrow$

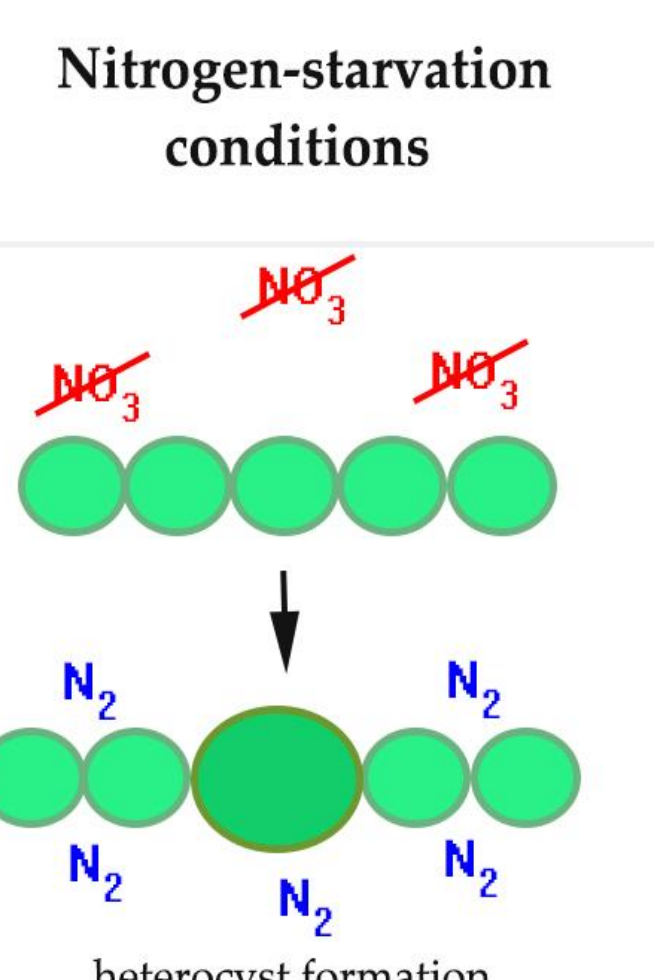

Diazotrophic-grow conditions

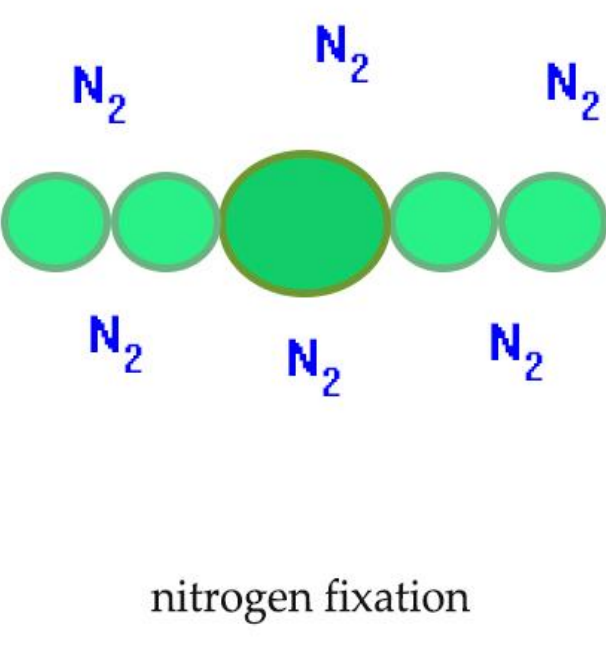

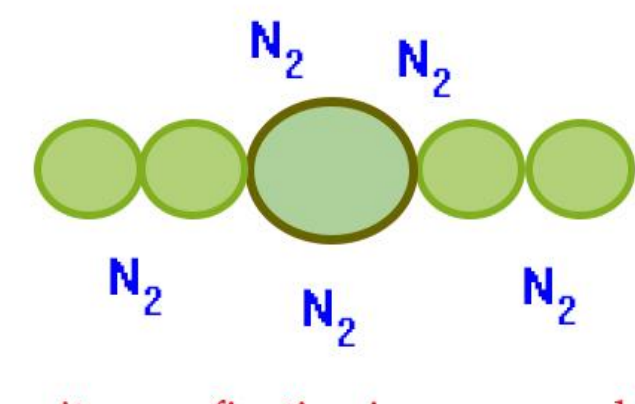

PII $\downarrow \quad n i f H \downarrow N i f D \downarrow N i f K \downarrow$

Fig. 4 . Nitrogen-fixing filamentous cyanobacteria Nostoc sp. in its normal state and after BMMA treatment under different nitrogen conditions.

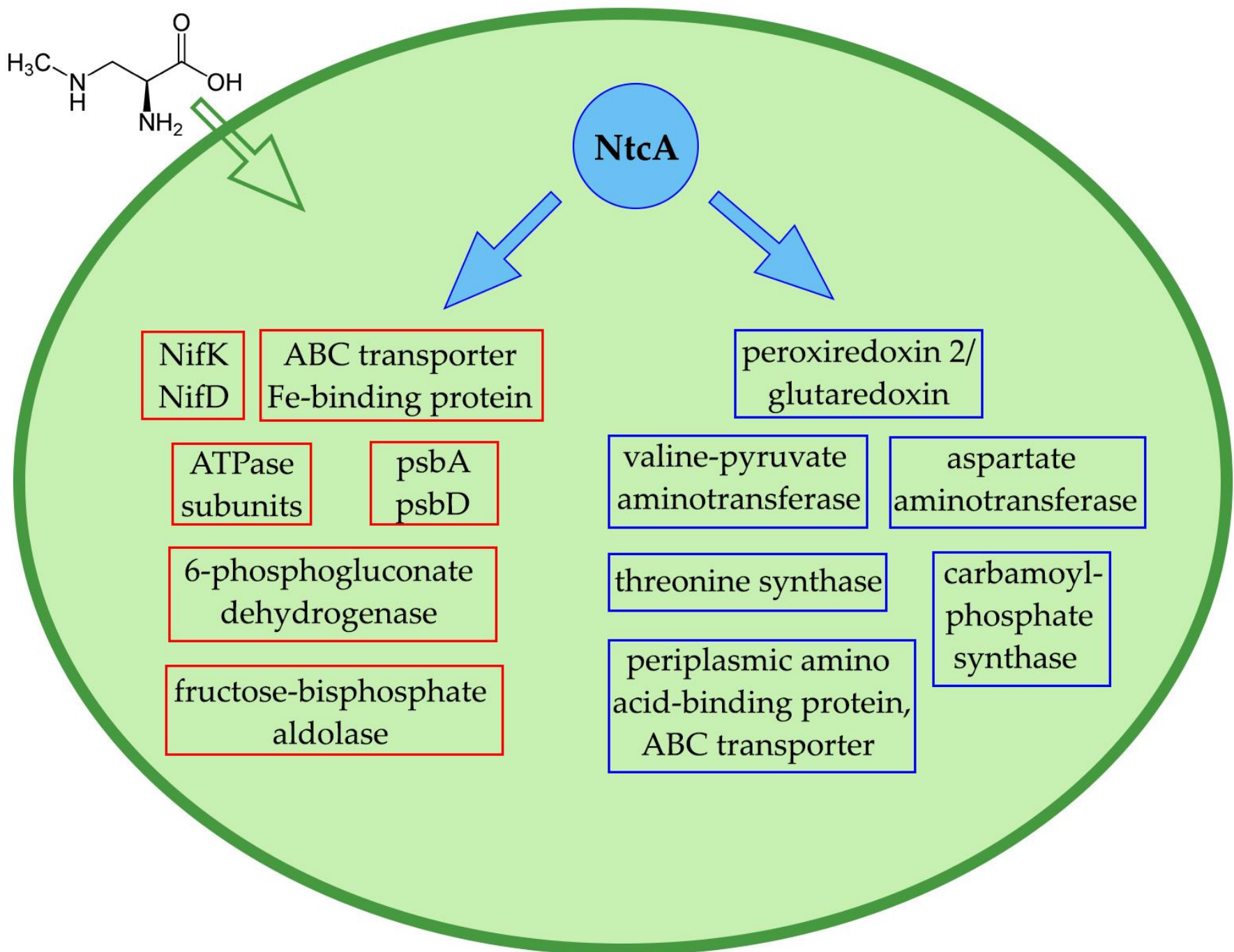

Fig.5. BMAA's effect on proteins encoded by genes that are under transcription control of the NtcA global regulator. Downregulated proteins are highlighted with a red frame and upregulated proteins are highlighted with a blue frame.

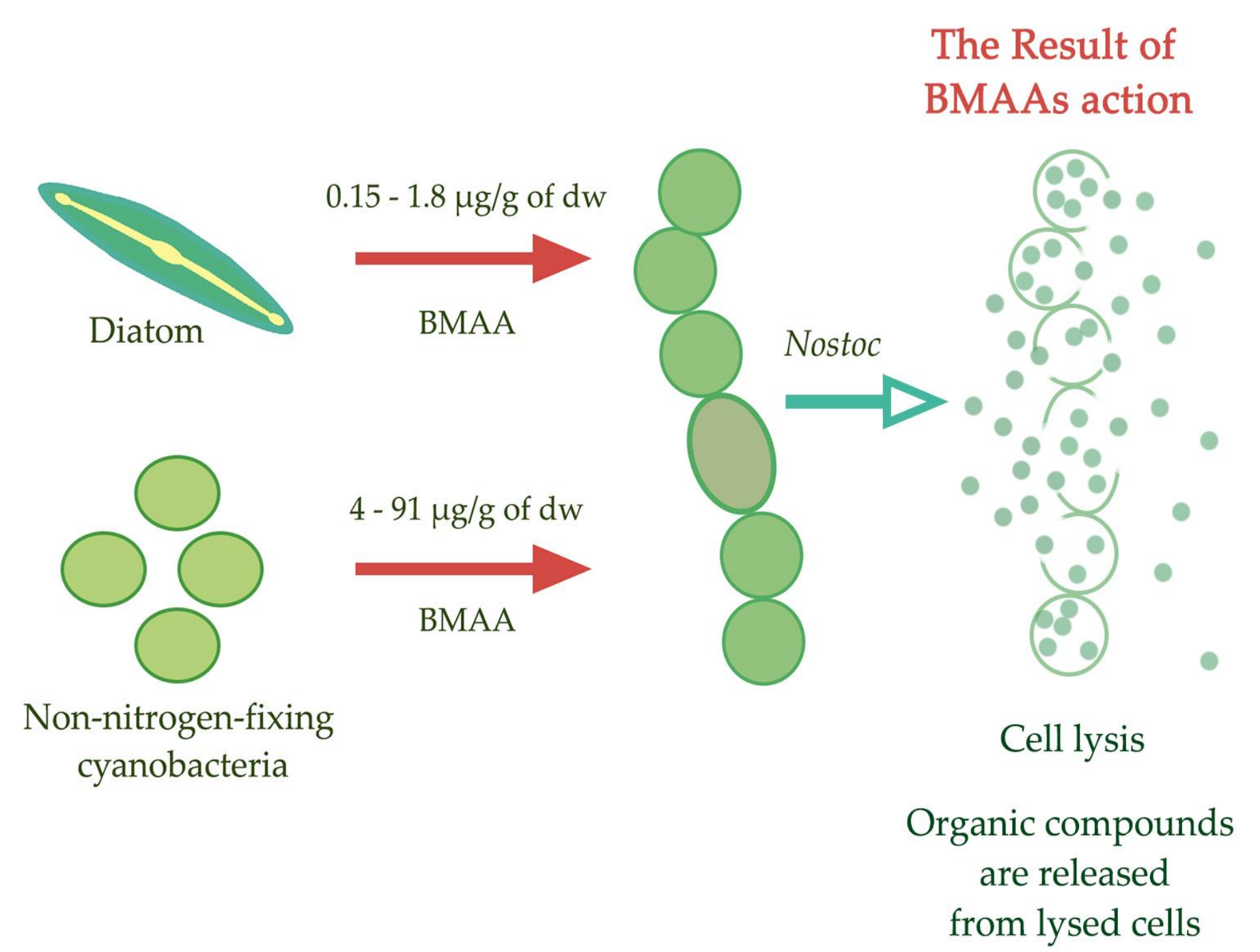

Fig.6. BMAA may be used by phytoplankton species as a possible allelopatic tool for control diazotrophic cyanobacteria cell populations during their strong competition for nitrogen and other nutrients. Nostoc cells undergo lysis under the presence of pxogenous BMAA (in relatively high amounts) and as a result: dissolved organic compounds that are necessary for the algae community nutrition are released.

\section{References}

Heimann K. Cirés S. 2015. Handbook of Marine Microalgae, 501-515. doi:10.1016/b978-0-12-800776-1.00033-9 2.Popova A. and Koksharova O. 2016. Biochemistry 2016, 81, 794-805, doi:10.1134/S0006297916080022 3. Berntzon L. et al. 2013. Mar. Drugs. 11, 3091-3108; doi:10.3390/md11083091.

4. Popova A. et al.2018. Environmental microbiology reports.Vol 10, № 3, pp. 369-377 DOI 10.1111/1758-2229.12647 5. Popova A. et al.2018. Toxins, 10, №11,p.478, DOI: 10.3390/toxins10110478

6. Koksharova O. et al. 2020. Toxins, 12, № 5, p. 310, doi:10.3390/toxins12050310

7. Koksharova O. et al. 2020. Toxins, 12, №6,p.372, DOI: 10.3390/toxins12060372.

8. Koksharova O. et al. 2020 (prepared for submission) 